\title{
A NOTE ON INPUT-OUTPUT SPECTRAL DENSITIES*
}

BY K. S. MILLER (Columbia University Electronics Research Laboratories)

If stationary noise $x(t)$ is applied to a linear time invariant system $\delta$ with transfer function $H(j \omega)$, then the mean-square value $\sigma^{2}$ of the output noise $z(t)$ is given by the well known formula

$$
\sigma^{2}=\int_{0}^{\infty}|H(j \omega)|^{2} w_{x}(f) d f
$$

where $w_{x}(f)$ is the spectral density of the input process $x(t)$. Apparently (1) has no simple analog when $x(t)$ is non-stationary. Of course, one can always write, even in the nonstationary case,

$$
\sigma^{2}(t)=\varepsilon z^{2}(t)=\int_{-\infty}^{\infty} \int_{-\infty}^{\infty} h(t-\zeta) h(t-\zeta) \psi_{x}(\xi, \zeta) d \xi d \zeta
$$

where $h(t)$ is the impulsive response of $S$ and $\psi_{x}(\xi, \zeta)=\varepsilon x(\xi) x(\zeta)$ is the correlation function of $x(t)$. However, in certain practical problems the formulation embodied in (1) is more convenient.

In this note we wish to deduce a formula similar to (1) in the case where the input $y(t)$ to $\delta$ is of the form

$$
y(t)=g(t) x(t)
$$

and $x(t)$ is stationary noise while the modulation function $g(t)$ is of the form

$$
g(t)=\sum_{n=1}^{N} a_{n} \cos \left(\omega_{n} t+\phi_{n}\right) .
$$

Such a situation may arise, for example, when a sinusoidal signal, corrupted by additive stationary noise, is applied to a square law detector followed by a linear filter s. If $K \cos \Omega t+\eta(t)$, where $\eta(t)$ is the noise, is applied to the square law detector, one of the terms in its output will be $(2 K \cos \Omega t) \eta(t)$-which is of the form (2). Thus, for example, if one wished to compute the signal-to-noise ratio at the output of the linear filter $\delta$, it would be necessary to compute (among other terms) the variance of the output of $\delta$ corresponding to the input $y(t)$. This is precisely the problem we have set ourselves.

Returning to (2) we see from the definition of $g(t)$, cf. (3), that $y(t)$ is non-stationary, but of special form. We shall show that if $z(t)$ is the output of $\delta$ corresponding to this input $y(t)$, then the mean-square value of the output noise $z(t)$ is

$$
\sigma^{2}(t)=\frac{1}{4} \int_{0}^{\infty}\left|M(\omega)+\{M(-\omega)\}_{c}\right|^{2} w_{x}(f) d f,
$$

where the subscript $C$ indicates the conjugate complex quantity,

$$
M(\omega)=\sum_{n=1}^{N} a_{n} H\left(j\left(\omega+\omega_{n}\right)\right) e^{i\left(\omega_{n} t+\phi_{n}\right)},
$$

${ }^{*}$ Received Nov. 19, 1962. This work in part was part of research directed by the Advanced Research Projects Agency of the Department of Defense and was administered by the Air Force Office of Scientific Research under Contract AF 49-(638)-1113. 
and $H(j \omega)$ is the transfer function of $\delta$. Certain generalizations can also be obtained.

We begin by writing the output noise $z(t)$ of $\delta$ arising from the input $y(t)$ as

$$
z(t)=\frac{1}{2 \pi} \int_{-\infty}^{\infty} H(j \omega) Y(j \omega) e^{j \omega t} d \omega
$$

where $Y(j \omega)$ is the Fourier transform of $y(t)$ :

$$
Y(j \omega)=\int_{-\infty}^{\infty} y(t) e^{-j \omega t} d t .
$$

A difficulty arises since $Y(j \omega)$ will not exist in the classical sense unless $g(t) e^{-i \omega t}$ is absolutely integrable - which it is not. This problem, as well as the subsequent use of the Dirac delta functional could be avoided. For example, we could replace $a_{n}$ in (3) by $a_{n} e^{-\alpha|t|}$ with $\alpha>0$, and then investigate the value of $\sigma^{2}$ as $\alpha$ approaches zero; or we could let $a_{n}=0$ for $|t|>T$ and investigate the value of $\sigma^{2}$ as $T$ increases without limit. However, it is more convenient to use the approach we employ below.

The mean-square value of the output noise is given by

$$
\sigma^{2}(t)=\varepsilon z^{2}(t)=\varepsilon \frac{1}{4 \pi^{2}} \int_{-\infty}^{\infty} \int_{-\infty}^{\infty} H(j \omega) H(j \beta) Y(j \omega) Y(j \beta) e^{j \omega t} e^{j \beta t} d \omega d \beta .
$$

Substituting the right-hand side of $(6)$ for $Y(j \omega)$ and $Y(j \beta)$ in (7) we obtain

$$
\sigma^{2}(t)=\frac{1}{4 \pi^{2}} \varepsilon \int_{-\infty}^{\infty} \int_{-\infty}^{\infty} \int_{-\infty}^{\infty} \int_{-\infty}^{\infty} H(j \omega) H(j \beta) y(\xi) y(\zeta) e^{-i \omega \xi} e^{-j \beta \zeta} e^{j \omega t} e^{i \beta t} d \omega d \beta d \xi d \zeta .
$$

Now,

$$
\varepsilon y(\xi) y(\zeta)=g(\xi) g(\zeta) \psi_{x}(\xi-\zeta)
$$

where $\psi_{x}$ is the correlation function of $x(t)$. Thus (8) may be written as

$$
\sigma^{2}(t)=\frac{1}{4 \pi^{2}} \int_{-\infty}^{\infty} \int_{-\infty}^{\infty} H(j \omega) H(j \beta) e^{j \omega t} e^{j \beta t} X(\omega, \beta) d \omega d \beta,
$$

where

$$
X(\omega, \beta)=\int_{-\infty}^{\infty} \int_{-\infty}^{\infty} g(\xi) g(\zeta) \psi_{x}(\xi-\zeta) e^{-i \omega \xi} e^{-i \beta \zeta} d \xi d \zeta
$$

Since

$$
g(\xi) g(\zeta)=\sum_{n=1}^{N} \sum_{m=1}^{N} a_{n} a_{m} \cos \left(\omega_{n} \xi+\phi_{n}\right) \cos \left(\omega_{m} \zeta+\phi_{m}\right)
$$

we may write

$$
X(\omega, \beta)=\sum_{n=1}^{N} \sum_{m=1}^{N} a_{n} a_{m} X_{n m}(\omega, \beta)
$$

where

$$
X_{n m}(\omega, \beta)=\int_{-\infty}^{\infty} \int_{-\infty}^{\infty} \cos \left(\omega_{n} \xi+\phi_{n}\right) \cos \left(\omega_{m} \zeta+\phi_{m}\right) \psi_{x}(\xi-\zeta) e^{-j \omega \xi} e^{-i \beta \zeta} d \xi d \zeta .
$$

If we make the change of variable $u=\xi-\zeta, v=\zeta$ with unity Jacobian in $X_{n m}(\omega, \beta)$, we obtain

$$
X_{n m}(\omega, \beta)=\int_{-\infty}^{\infty} \cos \left(\omega_{m} v+\phi_{m}\right) e^{-i v(\omega+\beta)} d v \int_{-\infty}^{\infty} \cos \left(\omega_{n} u+\omega_{n} v+\phi_{n}\right) \psi_{x}(u) e^{-i \omega u} d u .
$$


The second integral is readily evaluated as

$$
\begin{aligned}
\int_{-\infty}^{\infty} \cos \left(\omega_{n} u+\omega_{n} v+\phi_{n}\right) \psi_{x}(u) e^{-i \omega u} d u & \\
& =\frac{1}{4} w_{x}\left(f+f_{n}\right) e^{-i\left(\omega_{n} \nu+\phi_{n}\right)}+\frac{1}{4} w_{x}\left(f-f_{n}\right) e^{i\left(\omega_{n} v+\phi_{n}\right)},
\end{aligned}
$$

where $f=\omega / 2 \pi, f_{n}=\omega_{n} / 2 \pi, 1 \leq n \leq N$, and

$$
w_{x}(f)=2 \int_{-\infty}^{\infty} \psi_{x}(u) e^{-i \omega u} d u
$$

is the spectral density of $x(t)$. Substituting (12) in (11) we obtain

$$
\begin{gathered}
X_{n m}(\omega, \beta)=\frac{\pi}{4} w_{x}\left(f+f_{n}\right)\left[\delta\left(\omega+\beta+\omega_{n}-\omega_{m}\right) e^{i\left(\phi_{m}-\phi_{n}\right)}+\delta\left(\omega+\beta+\omega_{n}+\omega_{m}\right) e^{-j\left(\phi_{m}+\phi_{n}\right)}\right] \\
+\frac{\pi}{4} w_{x}\left(f-f_{n}\right)\left[\delta\left(\omega+\beta-\omega_{n}-\omega_{m}\right) e^{j\left(\phi_{m}+\phi_{n}\right)}+\delta\left(\omega+\beta-\omega_{n}+\omega_{m}\right) e^{-i\left(\phi_{m}-\phi_{n}\right)}\right] .
\end{gathered}
$$

From (10), we may write $\sigma^{2}$ [cf. (9)] as

$$
\begin{aligned}
\sigma^{2} & =\frac{1}{4 \pi^{2}} \sum_{n, m} a_{n} a_{m} \int_{-\infty}^{\infty} \int_{-\infty}^{\infty} H(j \omega) H(j \beta) e^{j \omega t} e^{i \beta t} X_{n m}(\omega, \beta) d \omega d \beta \\
& =\frac{1}{4 \pi^{2}} \sum_{n, m} a_{n} a_{m} Y_{n m},
\end{aligned}
$$

where

$$
Y_{n m}=\int_{-\infty}^{\infty} \int_{-\infty}^{\infty} H(j \omega) H(j \beta) e^{i \omega t} e^{j \beta t} X_{n m}(\omega, \beta) d \omega d \beta .
$$

Equation (13) may now be used to write $Y_{n m}$ as

$$
\begin{aligned}
& Y_{\text {um }}=\frac{\pi}{4} \int_{-\infty}^{\infty} \int_{-\infty}^{\infty} H(j \omega) H(j \beta) e^{j \omega t} e^{i \beta t} w_{x}\left(f+f_{n}\right)\left[\delta\left(\omega+\beta+\omega_{n}-\omega_{m}\right) e^{i\left(\phi_{m}-\phi_{n}\right)}\right. \\
&\left.+\delta\left(\omega+\beta+\omega_{n}+\omega_{m}\right) e^{-i\left(\phi_{m}+\phi_{n}\right)}\right] d \omega d \beta, \\
&+\frac{\pi}{4} \int_{-\infty}^{\infty} \int_{-\infty}^{\infty} H(j \omega) H(j \beta) e^{i \omega t} e^{i \beta t} w_{x}\left(f-f_{n}\right)\left[\delta\left(\omega+\beta-\omega_{n}-\omega_{m}\right) e^{i\left(\phi_{m}+\phi_{n}\right)}\right. \\
&+\left.\delta\left(\omega+\beta-\omega_{n}+\omega_{m}\right) e^{-i\left(\phi_{m}-\phi_{n}\right)}\right] d \omega d \beta .
\end{aligned}
$$

Replacing $\omega+\omega_{n}$ by $\omega$ in the first integral and $\omega-\omega_{n}$ by $\omega$ in the second integral enables us to write $Y_{n m}$ as

$$
\begin{aligned}
Y_{n m}=\frac{\pi}{4} \int_{-\infty}^{\infty} H(j(\omega- & \left.\left.\omega_{n}\right)\right) H\left(-j\left(\omega-\omega_{m}\right)\right) e^{i\left(\omega_{m}-\omega_{n}\right) t} w_{x}(f) d \omega e^{j\left(\phi_{m}-\phi_{n}\right)} \\
& +\frac{\pi}{4} \int_{-\infty}^{\infty} H\left(j\left(\omega-\omega_{n}\right)\right) H\left(-j\left(\omega+\omega_{m}\right)\right) e^{-i\left(\omega_{m}+\omega_{n}\right) t} w_{x}(f) d \omega e^{-j\left(\phi_{n}+\phi_{m}\right)} \\
& +\frac{\pi}{4} \int_{-\infty}^{\infty} H\left(j\left(\omega+\omega_{n}\right)\right) H\left(-j\left(\omega-\omega_{m}\right)\right) e^{i\left(\omega_{n}+\omega_{m}\right) t} w_{x}(f) d \omega e^{j\left(\phi_{m}+\phi_{n}\right)} \\
& +\frac{\pi}{4} \int_{-\infty}^{\infty} H\left(j\left(\omega+\omega_{n}\right)\right) H\left(-j\left(\omega+\omega_{m}\right)\right) e^{i\left(\omega_{n}-\omega_{m}\right) t} w_{x}(f) d \omega e^{-i\left(\phi_{m}-\phi_{m}\right)}
\end{aligned}
$$


This result and (14) then imply

$$
\begin{aligned}
\sigma^{2}=\frac{1}{4} \int_{0}^{\infty}[M(\omega) M(-\omega)+ & M(\omega)\{M(\omega)\}_{c} \\
+ & \left.\{M(-\omega)\}_{C} M(-\omega)+\{M(-\omega) M(\omega)\}_{c}\right] w_{x}(f) d f,
\end{aligned}
$$

which immediately reduces to (4).

If in place of equation (7) we start with

$$
\psi_{z}(t ; \tau)=\varepsilon z(t) z(t+\tau)
$$

and employ the Wiener-Khintchine relations ${ }^{1}$ to write

$$
w_{z}(t ; f)=2 \int_{-\infty}^{\infty} \psi_{z}(t ; \tau) e^{-i \omega \tau} d \tau
$$

as the time dependent spectral density, then using the above techniques we can show that

$$
\begin{array}{r}
w_{z}(t ; f)=\frac{1}{4} H(j \omega)\left\{\sum_{m=1}^{N} a_{m}\left[\left\{M\left(\omega-\omega_{m}\right)\right\}_{c}+M\left(-\omega+\omega_{m}\right)\right] e^{j\left(\omega_{m} t+\phi_{m}\right)} w_{x}\left(f-f_{m}\right)\right. \\
\left.+\sum_{m=1}^{N} a_{m}\left[\left\{M\left(\omega+\omega_{m}\right)\right\}_{c}+M\left(-\omega-\omega_{m}\right)\right] e^{-i\left(\omega_{m} t+\phi_{m}\right)} w_{x}\left(f+f_{m}\right)\right\} .
\end{array}
$$

It is easily seen, then, that

$$
\sigma^{2}=\frac{1}{2} \int_{-\infty}^{\infty} w_{z}(t ; f) d f
$$

where $\sigma^{2}$ is given by (4).

'D. G. Lampard, Generalization of the Wiener-Khintchine theorem to nonstationary processes, J. Appl. Phys. 25, 802-803(1954)

\section{Correction to the paper}

\section{DUALITY IN NONLINEAR PROGRAMMING}

Quarterly of Applied Mathematics, XX, 300-302 (1962)

By O. I. MANGASARIAN (Shell Development Company)

There is an incorrect statement of a previous result. In particular the last sentence of the Converse Duality Theorem should read:

"If $\varphi(x)$ is quadratic and if $g(x)$ is linear, then a weaker converse theorem is also true if $\varphi(x)$ is merely convex and twice continuously differentiable." 